\title{
Performed Study Administrative Activity
}

National Cancer Institute

\section{Source}

National Cancer Institute. Performed Study Administrative Activity. NCI Thesaurus. Code C93436.

The completed study level administrative activity that is independent of a study subject. 